\title{
Methionyl-tRNA synthetase inhibitor has potent in vivo activity in a novel Giardia lamblia luciferase murine infection model
}

\author{
Samantha A. Michaels ${ }^{a \#}$, Han-Wei Shih ${ }^{b \#}$, Bailin Zhang ${ }^{b}$, Edelmar D. Navaluna ${ }^{a}$, Zhongsheng \\ Zhang $^{\mathrm{c}}$, Ranae M. Ranadea, J. Robert Gillespie ${ }^{\mathrm{a}}$, Ethan A. Merrittc, Erkang Fan' ${ }^{\mathrm{c}}$, Frederick S. \\ Bucknera $^{\mathrm{a}}$ Alexander R. Paredez ${ }^{\mathrm{b} *}$ and Kayode K. Ojo ${ }^{\mathrm{a} *}$
}

aDepartment of Medicine, Division of Allergy and Infectious Disease, Center for Emerging and Reemerging Infectious Disease (CERID), University of Washington, Seattle, WA 98109 USA bDepartment of Biology, University of Washington, Seattle, WA 98195 USA 'Department of Biochemistry, University of Washington, Seattle, WA 98195 USA

\# Contributed equally

* Corresponding authors

${ }^{\star}$ Address correspondence to:

Alexander R. Paredez, Department of Biology, University of Washington, P.O. Box 351800 Seattle, WA 98195-1800 USA Phone 206-221-9197 Fax 206-543-3041 Email: aparedez@uw.edu

and

Kayode K. Ojo, Department of Medicine, Division of Allergy and Infectious Disease, Center for Emerging and Re-emerging Infectious Disease (CERID), University of Washington, Seattle, Washington 98109 USA

Phone 206-543-0821 Email: ojo67kk@u.washington.edu

Running Title: MetRS inhibitors prevent growth of luciferase G. lamblia strains 
Abstract

Objectives: Methionyl-tRNA synthetase (MetRS) inhibitors are under investigation for the treatment of intestinal infections caused by Giardia lamblia. To properly analyze the therapeutic potential of the MetRS inhibitor 1717, experimental tools including a robust cell-based assay and a murine model of infection were developed based on novel strains of G. lamblia that employ luciferase reporter systems to quantify viable parasites.

Methods: Systematic screening of Giardia-specific promoters and luciferase variants led to the development of a strain expressing the click beetle green luciferase. Further modifying this strain to express NanoLuc created a dual reporter strain capable of quantifying parasites in both the trophozoite and cyst stages. These strains were used to develop a high throughput cell assay and a mouse infection model. A library of MetRS inhibitors was screened in the cell assay and $\mathbf{1 7 1 7}$ was tested for efficacy in the mouse infection model.

Results: Cell viability in in vitro compound screens was quantified via bioluminescence readouts while infection loads in mice were monitored with noninvasive whole-animal imaging and fecal analysis. Compound 1717 was effective in clearing mice of Giardia infection in 3 days at varying doses, which is supported by data from enzymatic and phenotypic cell assays.

Conclusions: The new in vitro and in vivo assays based on luciferase expression by engineered G. lamblia strains are useful for the discovery and development of new therapeutics for giardiasis. MetRS inhibitors, as validated by 1717 , have promising anti-giardiasis properties that merit further study as alternative therapeutics. 
Giardia lamblia infections are among the most common causes of chronic diarrhea in children in resource-limited environments. New therapeutics are needed to address issues with existing therapies including resistance, toxicity, and reduced efficacy. No vaccines have been developed for clinical use, so case management depends solely on antimicrobial chemotherapy. ${ }^{1}$ Current therapies approved by the Food and Drug Administration for giardiasis include metronidazole and tinidazole. ${ }^{2-4}$ However, approximately $20 \%$ of clinical cases involve metronidazole- (and presumably tinidazole-) resistant Giardia. ${ }^{5}$ Second-line drugs such as albendazole, nitazoxanide, furazolidone, and paromomycin generally have lower efficacy rates and/or potentially dangerous side effects. ${ }^{6}$ All of these factors necessitate the development of a new therapeutic, which requires various experimental tools for screening and verification of efficacy of potential leads.

While experimental methods suitable for drug screening against G. lamblia have been described in literature, ${ }^{7}$ in vivo infection models in adult mice can benefit from an improved method of monitoring infection. This deficiency of the in vivo model can be explained in part by earlier studies showing instability in the expression of transgenes in Giardia compared to many other eukaryotic cells. ${ }^{8}$ The instability leads to variable expression as Giardia, by an unknown mechanism, downregulates transgenic markers. ${ }^{8}$ We recently described a luciferase-based reporter system in G. lamblia WBC6 trophozoites for quantitative fluorescence readouts from a red-shifted firefly luciferase reporter gene (PpyRE9h) under the control of the $\beta$-tubulin promoter (pßTub). ${ }^{9}, 10$ While the bioluminescent output of G. lamblia WBC6:p $\beta T u b:: P p y R E 9 h$ was sufficiently stable for 24-hour in vitro assays, it lacked long-term stability over multiple passages and proved unsuitable for the mouse model of infection. In this study, we describe the development of a new strain of G. lamblia using a click beetle green luciferase (CBG99) under a 
compound libraries and in vivo efficacy assays. A second strain was engineered by endogenously tagging one copy of the cyst wall protein 1 (CWP1) gene with NanoLuc (Nluc) in the G. lamblia WBC6:pGDHS::CBG99 strain, establishing a second developmentally induced reporter that specifically measures cyst quantity. The strains can be visualized and measured in an animal model of infection using noninvasive imaging to evaluate experimental drug effects in Giardiainfected animals. The developed mouse model was validated using the standard metronidazole treatment and subsequently was used to confirm that $G$. lamblia methionyl-tRNA synthetase (MetRS) enzyme inhibitor $\mathbf{1 7 1 7}$ is a potential therapeutic alternative for treatment of clinical giardiasis.

\section{Materials and Methods}

Plasmid construction and G. lamblia transfection: To optimize luminescence of Giardia for in vitro and in vivo experiments, various combinations of luciferase genes with Giardia-specific promoters were tested. Twelve gene constructs were generated: three containing PpyRE9h driven by three Giardia promoters, five containing various luciferase genes driven by the long glutamate dehydrogenase promoter (pGDHL), and four containing CBG99 driven by four Giardia promoters. The three PpyRE9h constructs were driven by $\mathrm{PGDHL}$, the short glutamate dehydrogenase promoter (pGDHS), ${ }^{11}$ and the ornithine carboxytranferase promoter (pOCT). ${ }^{12}$ These were amplified by PCR using the primers found in Table 1 and digested with EcoRI and Xbal. The promoter fragments were cloned into the EcoRI-Xbal site of integration vector pPACVinteg. ${ }^{10,13}$ For the luciferase systems driven by $\mathrm{pGDHL}$, the coding regions of the various luciferase genes were amplified with the primers from Table 1 and digested with Xbal and Pacl. The Xbal-Pacl fragments were cloned downstream of the promoter in the pPACV-integ vector's Xbal-Pacl cloning site. Selected luciferase genes included standard firefly luciferase (Fluc), firefly luciferase 2 (Luc2), red click beetle luciferase (CBR), enhanced green-emitting luciferase (Eluc), and CBG99. To generate the CBG99 constructs, the pGDHL, pGDHS, pOCT, and pßTub were 
117 amplified with primers from Table 1, digested using EcoRI and Xbal, and cloned into the EcoRI

118 and Xbal digested vector pPACV-integ. To generate the CWP1-NLuc construct, the CWP1 119 (GL50803_5638) and NLuc genes were amplified with the primers from Table 1 and digested with $120 \mathrm{Xbal}-\mathrm{BamHI}$ and BamHI-EcoRI, respectively, then cloned into the Xball and EcoRI digested pKS121 NEO vector.

For integration, $30 \mu \mathrm{g}$ of the pPACV-integ constructs was linearized with Swal overnight at $25^{\circ} \mathrm{C}$ while CWP1-NLuc/pKS-NEO construct was linearized with Stul overnight at $37^{\circ} \mathrm{C}$. They were then precipitated with ethanol and incubated with $300 \mu \mathrm{L}$ of chilled G. lamblia cells $\left(\sim 13 \times 10^{6}\right.$ cells $/ \mathrm{mL}$ ) for 30 minutes before electroporation (Bio-Rad GenePulser $\mathrm{X}$ at $375 \mathrm{~V}, 1000 \mu \mathrm{F}, 750$ ohms). After electroporation, cells were incubated on ice for 10 minutes, then transferred to fresh media at $37^{\circ} \mathrm{C}$. Transfectants were selected with puromycin after overnight recovery.

Parasite cultures: G. lamblia WBC6:pßTub::PpyRE9h and wild type (WBC6, ATCC 50803) trophozoites were the starting strains used in these studies. ${ }^{10}$ These and all the newly transfected strains were grown in TYI-S-33 medium supplemented with $10 \%$ bovine serum and $0.05 \mathrm{mg} / \mathrm{mL}$ bovine bile without (wild type WBC6) or with (transfected strains) antibiotic selection pressure of $32 \mu \mathrm{g} / \mathrm{mL}$ puromycin dihydrochloride (Gibco, Dublin, Ireland). ${ }^{10,14}$ Cultures were incubated at $37^{\circ} \mathrm{C}$ in $16 \mathrm{~mL}$ Falcon round bottom polystyrene test tubes (Corning Inc., Corning, NY).

G. lamblia trophozoite in vitro bioluminescence optimization: The optimum concentration of luminescent substrate, D-Luciferin (GoldBio, USA), required for cell identification was determined as earlier described. ${ }^{10}$ AkaLumine (a D-Luciferin analog that is not recognized by the CBG99 enzyme) was used as a control. ${ }^{15,16}$ Plates were incubated for 5, 10, 15, 30, 60, 90, 120, 150, and 180 minutes at room temperature. Parasite numbers were correlated with bioluminescence intensity by plating a two-fold serial dilution of cells. ${ }^{10}$ Plates were read with an EnVision Multilabel 140 Plate Reader (Perkin Elmer, USA) after incubation at room temperature. Assays were repeated on different days at least twice. 
G. Iamblia Iuciferase expression stability: To evaluate the stability of $G$. lamblia

WBC6:pGDHL::PpyRE9h and G. lamblia WBC6:pGDHL::CBG99 in the absence of puromycin

selection, the strains were diluted (1:130 dilutions) twice a week with or without puromycin for 4

weeks. The luciferase activity proportional to cell concentrations was measured as described above.

G. lamblia cell assay development: Assays were performed in clear, flat bottom 96- or 384-well plates (Corning Inc., Corning, NY) in TYDK media. ${ }^{10}$ Compound screens used one concentration between $20 \mu \mathrm{M}$ and $1 \mu \mathrm{M}$, and dose response assays employed three-fold serial dilutions starting at concentrations up to $60 \mu \mathrm{M}$, depending on the particular compound's potency. All experimental wells were repeated in duplicate or quadruplicate within the same plate. Compounds were incubated with $250,000 \mathrm{G}$. lamblia trophozoites $/ \mathrm{mL}$ at a final DMSO concentration of $\leq 0.33 \%$. Negative (DMSO) and positive (Metronidazole) controls were included in each assay. The plates were placed in BD GasPak EZ anaerobe pouches (Becton Dickinson, San Jose, California) and incubated at $37^{\circ} \mathrm{C}$ for 44 hours. Growth was evaluated under a microscope before vinyl stickers were added to the bottom to prepare for luminescence reading. Ten $\mu \mathrm{L}$ of $2.5 \mathrm{mg} / \mathrm{mL} \mathrm{D}$-Luciferin was added to each well and the reaction was incubated at room temperature for 5 minutes on a shaker protected from light. Luminescence was read with an EnVision Plate Reader. ${ }^{10}$ Response curves and $\mathrm{EC}_{50}$ values (the concentration at which cell growth is inhibited by $50 \%$ ) were calculated using GraphPad Prism 6 (GraphPad, LaJolla, CA). All assays were repeated on 161 different days.

Using the G. lamblia WBC6:pGDHS::CBG99 strain, a library of MetRS inhibitors was initially screened at a single concentration of $20 \mu \mathrm{M}$. G. lamblia WBC6:pGDHS::CBG99 and WBC6:pGDHS::CBG/Nluc trophozoites were used to confirm hits in a $2 \mu \mathrm{M}$ screen, where hits were identified as those causing $>80 \%$ inhibition of growth. 
Giardia murine model development and efficacy of 1717: Eight- to twelve-week old female

BALB/c mice weighing approximately $20 \mathrm{~g}$ were used for this study. To promote parasite

colonization, mice were administered antibiotics $(0.25 \mathrm{mg} / \mathrm{mL}$ ampicillin and neomycin) in drinking

water for the duration of the experiment starting 6 days before infection. ${ }^{17} \mathrm{~A}$ cocktail containing

ampicillin, neomycin and metronidazole at concentrations of $50 \mathrm{mg} / \mathrm{kg}, 15 \mathrm{mg} / \mathrm{kg}$ and $50 \mathrm{mg} / \mathrm{kg}$,

respectively, was dosed by oral gavage 3 days before infection. A second antibiotic dose

containing $50 \mathrm{mg} / \mathrm{kg}$ ampicillin and $15 \mathrm{mg} / \mathrm{kg}$ neomycin was given 1 day before infection. Mice

were administered $1 \times 10^{7}$ G. lamblia WBC6:pGDHS::CBG99 trophozoites in PBS by oral gavage.

Establishment of infection was confirmed using a noninvasive IVIS imaging method in which mice

were given an intraperitoneal injection of D-Luciferin $(150 \mathrm{mg} / \mathrm{kg})$, sedated using isoflurane and placed in the IVIS instrument with nose cones administering continuous anesthetic. ${ }^{17}$

A pilot in vivo drug treatment analysis with metronidazole at $50 \mathrm{mg} / \mathrm{kg}$ once a day (QD) as

a positive control and dosing vehicle (3\% ethanol and $7 \%$ Tween 80 in normal saline) as a

negative control was used for validation of the infection model. This was followed by other pilot

studies with 1717 at $50 \mathrm{mg} / \mathrm{kg}$ once a day (QD) and dosing vehicle controls to further optimize the

model. Once the model was functionally optimized, the following round of experiments tested

various doses of 1717: $50 \mathrm{mg} / \mathrm{kg}$ twice a day (BID), $25 \mathrm{mg} / \mathrm{kg}$ BID, and $50 \mathrm{mg} / \mathrm{kg}$ QD. Control

groups included treatment with dosing vehicle and treatment with metronidazole at $50 \mathrm{mg} / \mathrm{kg}$ BID.

Treatments were introduced by oral gavage for 3 days. The course of infection was tracked by 185 whole-animal imaging.

Fecal samples from individual mice were collected before and throughout the treatment timeline. DNA was extracted from the samples using the QIAamp Fast DNA Stool Mini Kit (Qiagen, Germany). Cyst shedding in treated and untreated mice was verified by PCR analysis of the highly 
G. lamblia cyst in vitro bioluminescence: An encystation protocol was adapted from

previous studies ${ }^{19,20}$ to demonstrate the signal intensity of $G$. lamblia WBC6:pGDHS::CBG/Nluc

cysts. Confluent trophozoites were incubated with encystation media for 48 hours, then pelleted

and resuspended in growth media for 24 hours. Cells were pelleted and resuspended in cold

deionized water and stored at $4^{\circ} \mathrm{C}$ overnight. The amount of luciferase-induced light emission was

quantified as described above, with the modification that Nano-Glo®luciferase reagent (Promega,

Madison, $\mathrm{WI}$ ) was the luminescent substrate.

\section{Results}

Promoter and luciferase gene selection: We previously used the $\beta$-tubulin promoter to drive

red-shifted firefly luciferase PpyRE9h in G. lamblia WBC6 cells as a tool to assay growth inhibition

by compounds in the MMV Pathogen Box. ${ }^{10}$ Further analysis showed that the luminescent signal

of PpyRE9h diminished after three weeks of continuous incubation with or without antibiotic 202

selection. To improve the expression level of PpyRE9h, the $p \beta T$ Tub was replaced by three Giardia constitutive promoters: a 206 bp pOCT, ${ }^{12}$ a 44 bp pGDHS, ${ }^{11}$ and a 165 bp pGDHL. ${ }^{11}$ The resultant

G. lamblia trophozoites expressing pGDHL::PpyRE9h and pOCT::PpyRE9h had two- and three-

fold increased luminescence expression levels relative to $p \beta T u b:: P p y R E 9 h$, respectively. There

was no significant change for pGDHS::PpyRE9h relative to the p $\beta T u b:: P p y R E 9 h$ strains.

However, the highest luminescence value that any combination of promoter and PpyRE9h

achieved was a modest 1200 photons/sec for the G. lamblia WBC6:pOCT::PpyRE9h strain 209

(Figure 1A). We speculated that this would not be bright enough for use in a mouse model of infection, where the luminescent signal must penetrate multiple layers of tissue to visualize 211 infection noninvasively. There also appeared to be a fitness cost for cells with the pOCT-driven 212 luciferase exhibited by a decrease in growth rate. Thus, further work was needed to establish a 213 brighter and more stable luciferase reporter system in G. lamblia. Earlier studies had 
214 demonstrated that Fluc, Luc2, and CBR had higher sensitivity than PpyRE9h to report promoter 215 activity in other systems. ${ }^{21,22}$ Hence, these red luciferases were assayed in Giardia.

The red luciferase genes PpyRE9h, Fluc, Luc2, and CBR inserted downstream of pGDHL

were transfected into G. lamblia WBC6 cells. Among the resultant strains, trophozoites with the 218 Fluc gene had the brightest signal intensity, followed by those with the Luc2 gene (Figure 1B). 219 Two green luciferase emitting genes, CBG99 and ELuc, previously shown to exhibit higher levels 220 of bioluminescence than Fluc, ${ }^{23,}{ }^{24}$ were also assayed for higher signals under the control of pGDHL. In our study, G. lamblia WBC6:pGDHL::CBG99 was 100-fold brighter in signal intensity than G. lamblia WBC6:pGDHL::Eluc. We conclude that G. lamblia WBC6:pGDHL::CBG99 is the brightest luciferin-based reporter system among the red and green luciferases tested (Figure 1B).

subsequently performed. The CBG99 gene in the transfection construct was placed under the

control of four different Giardia promoters: $p \beta T u b, p O C T, p G D H S$ and $p G D H L$. All the transgenic

G. lamblia WBC6 strains expressing CBG99 luciferase gave robust bioluminescence signals, with pGDHS::CBG99 being the lowest even on the log scale (Figure 1C). However, G. lamblia strains expressing the CBG99 luciferase driven by pßTub, pOCT and pGDHL promoters have substantial fitness disadvantages in their rate of growth and proliferation relative to the wild type strain. The

effect was least pronounced with the G. lamblia WBC6:pGDHS::CBG99 (Figure 1D). Though it had the lowest signal of the promoters tested with CBG99, the signal intensity was still several 233 fold brighter than the one given by pGDHS::PpyRE9h, and therefore it was more suitable for the drug screening assays and murine model development. was achieved after 5 minutes of incubation with D-Luciferin at a final concentration of $\sim 83 \mu \mathrm{g} / \mathrm{mL}$. At this concentration and incubation time, the relative light units signal was high enough to 
minutes after the addition of substrate, but the luminescence decreased at longer incubation

times. The amount of CBG99-driven bioluminescence activity was directly proportional to the

number of viable transfected G. lamblia trophozoites per well, as determined by a comparative

analysis with the cell count of transfected strains (Figure 2A). The blank media control wells showed no luminescence as compared to the empty background wells and to the sample wells.

AkaLumine showed no signal at any time point, as expected. The average Z' of this assay was

0.6 .

G. lamblia luciferase expression stability: In our previous study, the bioluminescent signal of

G. lamblia WBC6:pßTub::PpyRE9h was the same as the wild type G. lamblia WBC6 after three

weeks of incubation, suggesting a loss of the luciferase gene activity. Therefore, we tested for

signal stability in the G. lamblia WBC6:pGDHS::CBG99 cells in the absence of the selection

antibiotic, puromycin. After four weeks without antibiotic, the bioluminescent signal of G. lamblia

WBC6:pGDHS::CBG99 was detected in sufficiently high levels to be called stable expression.

The signal was 35 times brighter than that given by G. lamblia WBC6:pßTub::PpyRE9h cells after

the same amount of time, which shows no differentiable signal compared to the wild type (Figure

2B). This is especially important to guarantee robust detection of the bioluminescent signal for an

in vivo experimental model of chronic infection lasting over 3 weeks.

Screening of MetRS inhibitors including compound 1717: The initial MetRS inhibitor screen

at $20 \mu \mathrm{M}$ proved to be highly potent against the parasites, with inhibition rates $>98 \%$ across most

compounds. The concentration was subsequently dropped to $2 \mu \mathrm{M}$, where most compounds still

had potent inhibition (Figure 3A, Table 2). Compounds BKI1708 and BKI1770, which are highly

selective inhibitors of apicomplexan calcium dependent protein kinases not found in G. lamblia,

BKI1770 at both concentrations demonstrate the specificity of the assay. Percentage inhibition 
264 The $2 \mu \mathrm{M}$ screen in 384-well plates with G. lamblia WBC6:pGDHS::CBG99 and G. lamblia WBC6:pGDHS::CBG/Nluc had average Z' values of 0.7 and 0.6, respectively. The screening data shows that these compounds exhibit a similar inhibition profile against both strains (Figure 3A). Compound $\mathbf{1 7 1 7}$ has previously shown potent inhibition on Giardia MetRS enzyme (GMetRS) activity and its $\mathrm{EC}_{50}$ was determined to be $453 \mathrm{nM}^{26}$ The $\mathrm{EC}_{50}$ of 1717 for G. lamblia WBC6:pGDHS::CBG99 and WBC6:pGDHS::CBG/Nluc was found to be 465 nM and 392 nM, respectively (Figure $3 \mathrm{~B}$ ). The metronidazole $\mathrm{EC}_{50}$ values of $2.867 \mu \mathrm{M}$ and $3.114 \mu \mathrm{M}$ obtained for G. lamblia WBC6:pGDHS::CBG99 and WBC6:pGDHS::CBG/Nluc strains, respectively, are within the range of previously reported literature values. ${ }^{10,26-28}$

Efficacy of metronidazole and 1717 in the Giardia murine model: We describe here the development and evaluation of a mouse infection model as a tool for measuring growth and proliferation of parasites in experimental drug treatment assays. Infection in BALB/c female mice was established within 5 days as determined by IVIS imaging and held for more than two weeks while bacterial antibiotics were maintained in drinking water. The rate of G. lamblia WBC6:pGDHS::CBG99 infection of BALB/c female mice was around 90\%, which is consistent with earlier reports. ${ }^{29}$ IVIS image analysis revealed infection signals of up to 100 -fold higher radiance values than background luminescence from uninfected mice. metronidazole, the current available therapeutic. At $50 \mathrm{mg} / \mathrm{kg} \mathrm{QD}$, it delivered a cure after 4 days. In the subsequent round of experiments, the efficacy of compound 1717 was determined. 1717 showed no adverse effects during treatment and all mice were cleared of the infection after 3 days of dosing with $50 \mathrm{mg} / \mathrm{kg}$ BID, $25 \mathrm{mg} / \mathrm{kg} \mathrm{BID}$, and $50 \mathrm{mg} / \mathrm{kg}$ QD. Imaging $24 \mathrm{hr}$ after the final dose and one week after the final dose showed that the drug cleared the infection compared to the vehicle controls (Figure 4). A cure, in this case, is defined as a lack of visible luminescence above 
289 after the last dose and a negative reading from the stool PCR. ${ }^{30}$ Molecular detection analysis with 290 PCR confirmed the absence or presence of Giardia cysts expelled in the feces of treated and 291 untreated mice. All procedures involving animals were conducted in adherence to federal 292 regulations and University of Washington's Institutional Animal Care and Use Committee (IACUC) 293 guidelines.

G. Iamblia WBC6:pGDHS::CBG99 versus WBC6:pGDHS::CBG/NIuc: To further confirm infection and clearance during in vivo assays, we attempted to measure luminescence in $G$. lamblia WBC6:pGDHS::CBG99 cysts from fecal samples. There was no detectable luminescence even in our untreated infected controls. This is likely because the cyst wall, which protects cysts from rupturing in water, prevents the uptake of D-Luciferin. To improve upon this reporter strain, we endogenously tagged the CWP1 gene of the G. lamblia WBC6:pGDHS::CBG99 strain with Nluc. A plot of bioluminescence outputs from cysts of G. lamblia WBC6:pGDHS::CBG99 versus WBC6:pGDHS::CBG/Nluc using NanoGlo is presented in Figure 5A. The growth and proliferation 302 rate of the 2 strains was experimentally compared and shown to be similar (Figure 5B).

\section{Discussion and Conclusion}

Diarrheal syndromes are among the leading causes of morbidity and mortality by infectious

diseases. G. lamblia, a significant contributor to the overall global diarrheal burden, ${ }^{31}$ poses a

public health challenge due to diminishing treatment options. The luminescent G. lamblia strains described here could be an important tool in the search for new giardiasis treatments. G. lamblia WBC6:pGDHS::CBG99 and WBC6:pGDHS::CBG/Nluc were developed by a process of iterative refinement that led to the selection of the most suitable luciferase and promoter combination. The 311 bioluminescence signal remained stable for weeks in vitro and in vivo without concurrent 312 administration of puromycin to maintain selective pressure. We earlier determined that Nluc is 
314 Since mice shed relatively few cysts during infection, a more sensitive luciferase is required to 315 validate infection or clearance in experimental drug studies, which makes Nluc a good choice. 316 CBG99 is a better cell viability reporter as it requires the presence of ATP for luminescence and 317 therefore will not produce light in dead cells. That Nluc does not require ATP for luminescence 318 makes it a viable reporter for the cyst stage when cells are relatively dormant. Hence, Nluc was not considered as an alternative to CBG99 but rather as a supplement. Additionally, fusing Nluc to CWP1, which is secreted to form the cyst wall, eliminates the need for luminescent substrate 321 to travel through the cyst wall barrier in order to reach the luciferase. This modification will provide 322 the ability to follow total parasite load in animals as well as easily quantify cyst production.

We demonstrated the usefulness of the luciferase reporter system to evaluate treatment with compound $\mathbf{1 7 1 7}$ in a mouse model of giardiasis using the noninvasive IVIS Spectrum optical imaging system. Compound 1717, a fluoro-imidazopyridine, is one of a new class of inhibitors that stop protein synthesis by targeting parasitic MetRS as previously described. ${ }^{26}$ These inhibitors are lethal to $G$. lamblia parasites but nontoxic to mammalian cells in cell based assays. ${ }^{26}$ Chemical synthesis, pharmacokinetics, cytotoxicity, and inhibitory activity of compound 1717 on the wild type G. lamblia WBC6 strain and the GMetRS enzyme were previously described. ${ }^{26,32,33}$ Analysis of 1717's pharmacokinetic profile showed that a single $50 \mathrm{mg} / \mathrm{kg}$ oral dose in mice would have sufficient gut and systemic levels to be effective for treatment of mouse giardiasis. ${ }^{33}$ The compound is about 8 times more potent than metronidazole, which has an $\mathrm{EC}_{50}$ of $5 \mu \mathrm{M} .^{26}$ It has 333 solubilities of $52 \mu \mathrm{M}$ at $\mathrm{pH} 7.4,96 \mu \mathrm{M}$ at $\mathrm{pH} 6.5$ and $100 \mu \mathrm{M}$ at pH 2. Earlier experiments suggest that MetRS inhibitor 1717 has a "cidal" anti-Giardia activity. ${ }^{26}$ All of these factors lead to the use of 1717 in the present study as a potential therapeutic. Our results further support 1717, as its efficacy in clearing Giardia infections in mice has been demonstrated. Compound 1717, developed as a trypanosomal agent, was used as a proof of principle molecule to demonstrate 
339 therapeutics. Further development of this series of inhibitors could facilitate innovative therapeutic

340 options for Giardiasis whose etiologic agent, G. lamblia, is rapidly evolving past currently available 341 therapies. Since there is no overlap in mechanism of action with any currently available drugs, 342 cross-resistance is unlikely.

\section{Acknowledgement}

346 The authors would like to thank Dr. Kelly M. Hennessey, Matthew A. Hulverson, Nora Molasky, 347 Wesley C. Van Voorhis, and Ryan Choi for helpful discussions.

\section{Funding}

349 This study was supported by grants from National Institute of Allergy and Infectious Diseases and 350 National Institute of Child Health and Human Development under award numbers R01Al110708,

353 None to declare. 
Tables

Table 1. List of primers used in this study.

Sequence (5'-3')

OCT promoter

Forward

TTGCGGCCGCGAATTCTCAATTGTCAGCTTCATTTTTATTAT

Reverse

TTTCTAGATTTAATTTTCAGCCTCTACTGTAGAGCGTTTA

370

371

372

373

374

375

376

377

378

379

380

381

382

383

384

385

386

387

388

389

390

391

392

393

394

395

396

397

398

399

400

401

402

403

404

405

406

407

\section{Short GDH promoter}

Forward TTGAATTCGACCACAAATAACGCCTTTAA

Reverse TTTCTAGATTTAAAATCTGGGGCGCCTGTAATTAAA

\section{Long GDH promoter}

Forward TTGCGGCCGCGAATTCAATGTCCACAAACTAAAAGTATAC

Reverse TTTCTAGATTTAAAATCTGGGGCGCCTGTAATTAAA

\section{Fluc}

Forward

AATCTAGAATGGAAGACGTCAAAAACATAAA

Reverse

GCTTAATTAATTATTCTCGAGACACGGCGATCTTT

Luc2

Forward

AATCTAGAATGGAAGATGCCAAAAACATTAAGAA

Reverse

GCTTAATTAATTACACGGCGATCTTGCCGCCCTTCTT

CBR

Forward

AATCTAGAATGGTAAAGCGTGAGAAAAATGTCATCTATGG

Reverse

GCTTAATTAATTATTTGTACAAACCGCCGGCCTTCACCAA

Eluc

Forward

AATCTAGAATGGAGAGAGAGAAGAACGTGGTGTA

Reverse

GCTTAATTAACTACACATTGATCCTAGCAGAAGCACA

\section{CBG99}

Forward

TTTCTAGAATGGTGAAGCGTGAGAAAAATGTCATCTATGG

Reverse GGTTAATTAATTATTTGTACAAACCGCCGGCCTTCTCCA

\section{CWP1}

Forward

GCTCTAGACAACGGCTTACTAAATCATTCT

Reverse

CCGGATCCactagtAGGCGGGGTGAGGCAGTACTCTCC

\section{NanoLuc}

Forward

GTGGATCCGGAGGCGGTTCAGGCGGAGGTGGCTCTGTCTTCACACTCGAAG ATTTCGTT

Reverse

AAGAATTCTTACGCCAGAATGCGTTCGCACAGCCGC 
bioRxiv preprint dol: https://doi.org/10.1101/822924; this version posted October 29, 2019. The copyright holder for this preprint (which was not certified by peer review) is the author/funder, who has granted bioRxiv a license to display the preprint in perpetuity. It is made available under aCC-BY-ND 4.0 International license.

408 Table 2: Percentage inhibition of luciferase G. lamblia cells by MethS inhibitors showed similar inhibition 409 profile for the CBG99 and CBG99/Nluc strains

Percentage inhibition

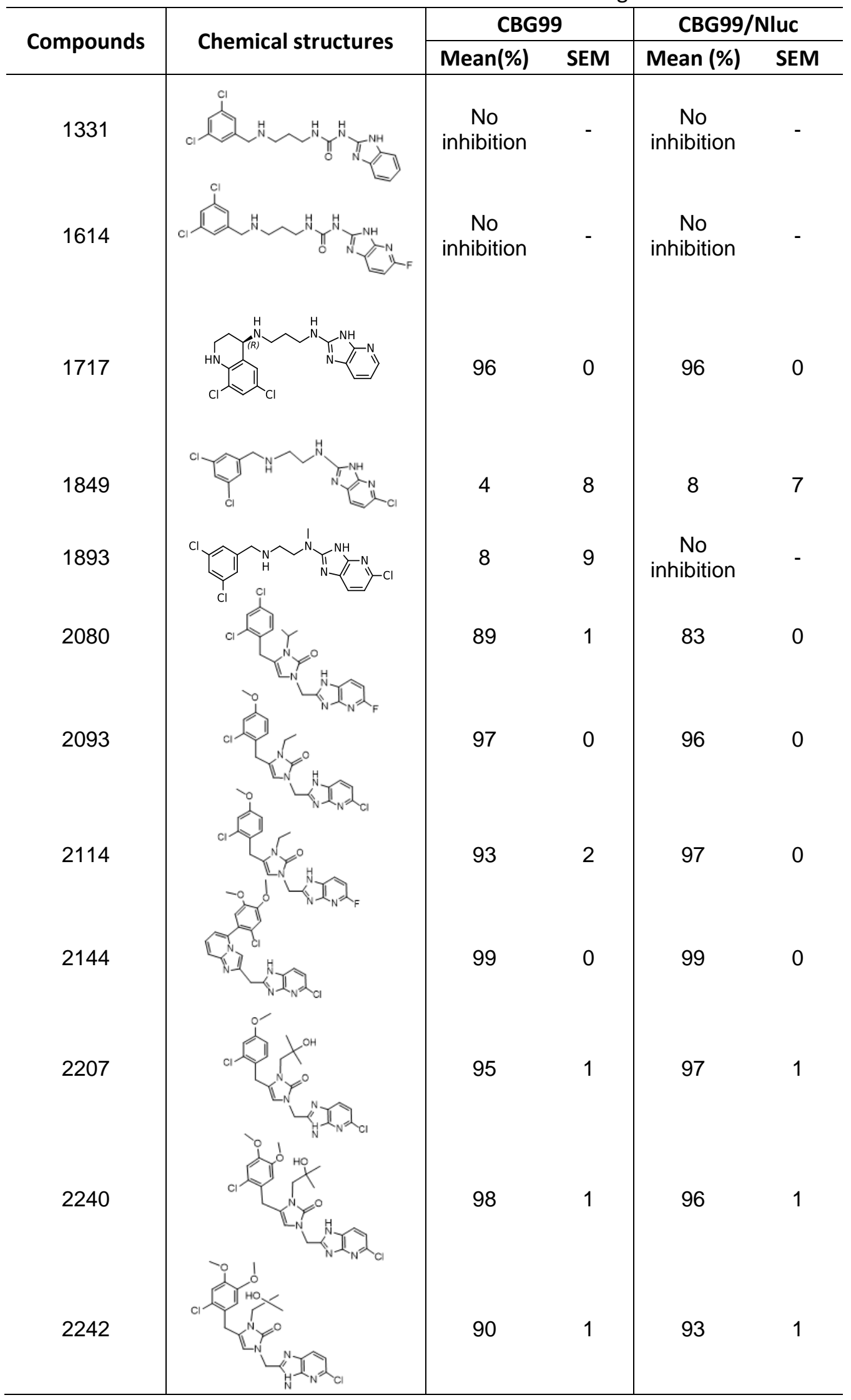


bioRxiv preprint doi: https://doi.org/10.1101/822924; this version posted October 29, 2019. The copyright holder for this preprint (which was not certified by peer review) is the author/funder, who has granted bioRxiv a license to display the preprint in perpetuity. It is made available under aCC-BY-ND 4.0 International license.

411 Figure 1: In vitro characterization of luciferase sensitivity.

A

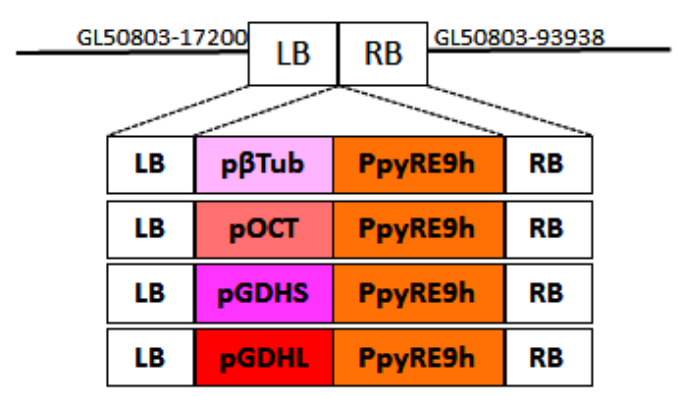

B

Giardia chromosome 5

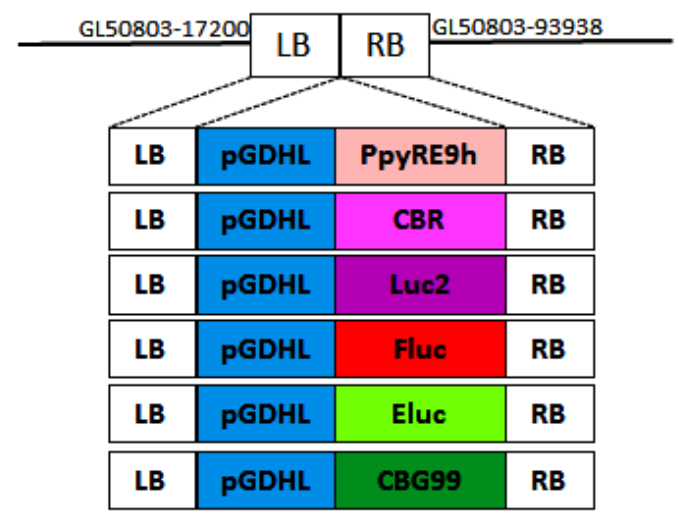

C

Giardia chromosome 5

\begin{tabular}{|c|c|c|c|}
\hline \multicolumn{1}{|l|}{ LB } & PBTUb & CBG99 & RB \\
\hline \hline LB & POCT & CBG99 & RB \\
\hline LB & PGDHS & CBG99 & RB \\
\hline LB & PGDHL & CBG99 & RB \\
\hline
\end{tabular}

412

413
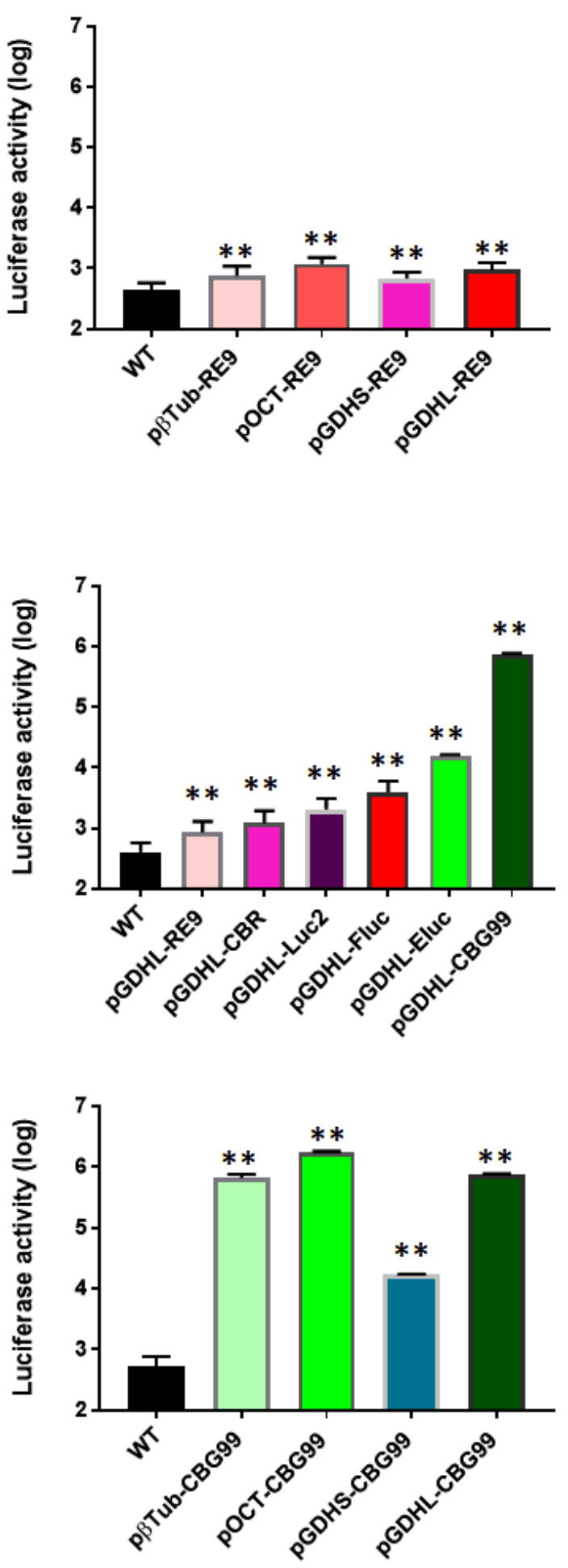


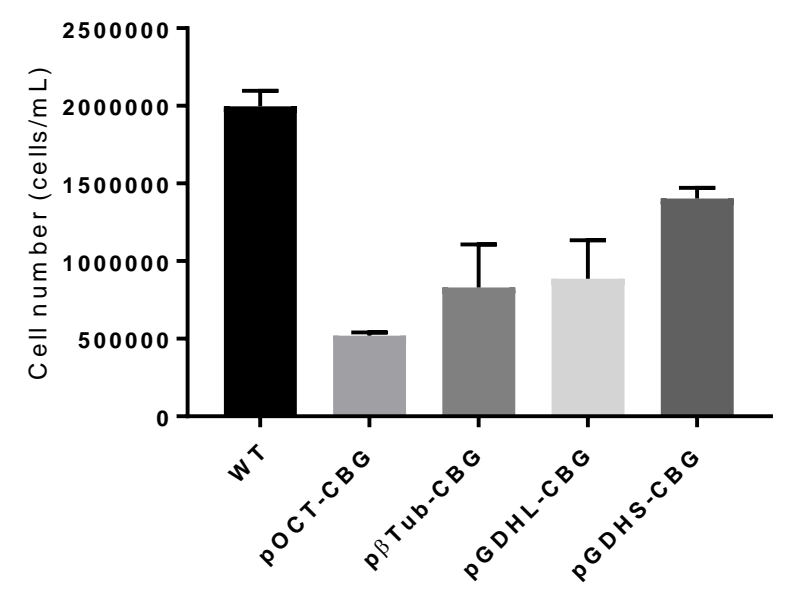

417 Figure 1. In vitro characterization of luciferase sensitivity. (a) Left: Schematic of PpyRE9h 418 driven by four Giardia specific promoters and homologous recombination. LB=left border, $419 \mathrm{RB}=$ right border. Right: In vitro comparison of PpyRE9h-dependent photon flux driven by four 420 Giardia specific promoters. (b) Left: Schematic of six different luciferases driven by PGDHL and 421 homologous recombination. Right: In vitro quantitative analysis of luciferase-dependent photon 422 flux from six different luciferases driven by pGDHL. (c) Left: Schematic of CBG99 driven by four 423 Giardia specific promoters and homologous recombination. Right: In vitro comparison of CBG99424 dependent photon flux driven by four Giardia specific promoters. (d) Effect of different promoters 425 on growth rate. At day $1,150 \mu \mathrm{L}\left(1 \times 10^{5}\right.$ cells $\left./ \mathrm{mL}\right)$ of culture was transferred to $13 \mathrm{~mL}$ TYDK. Cell density measurement after 3 days incubation at $37^{\circ} \mathrm{C}$ showed a significant fitness loss for most of the luciferase strains relative to the parent wild type parasite. G. lamblia WBC6:pGDHS::CBG99 showed the lowest fitness disadvantage. 


\section{Figure 2: Parasite cell count vs relative light units (RLU)}

W BC $6:$ :P DHS::CBG99 Trophozoite Lum inescence
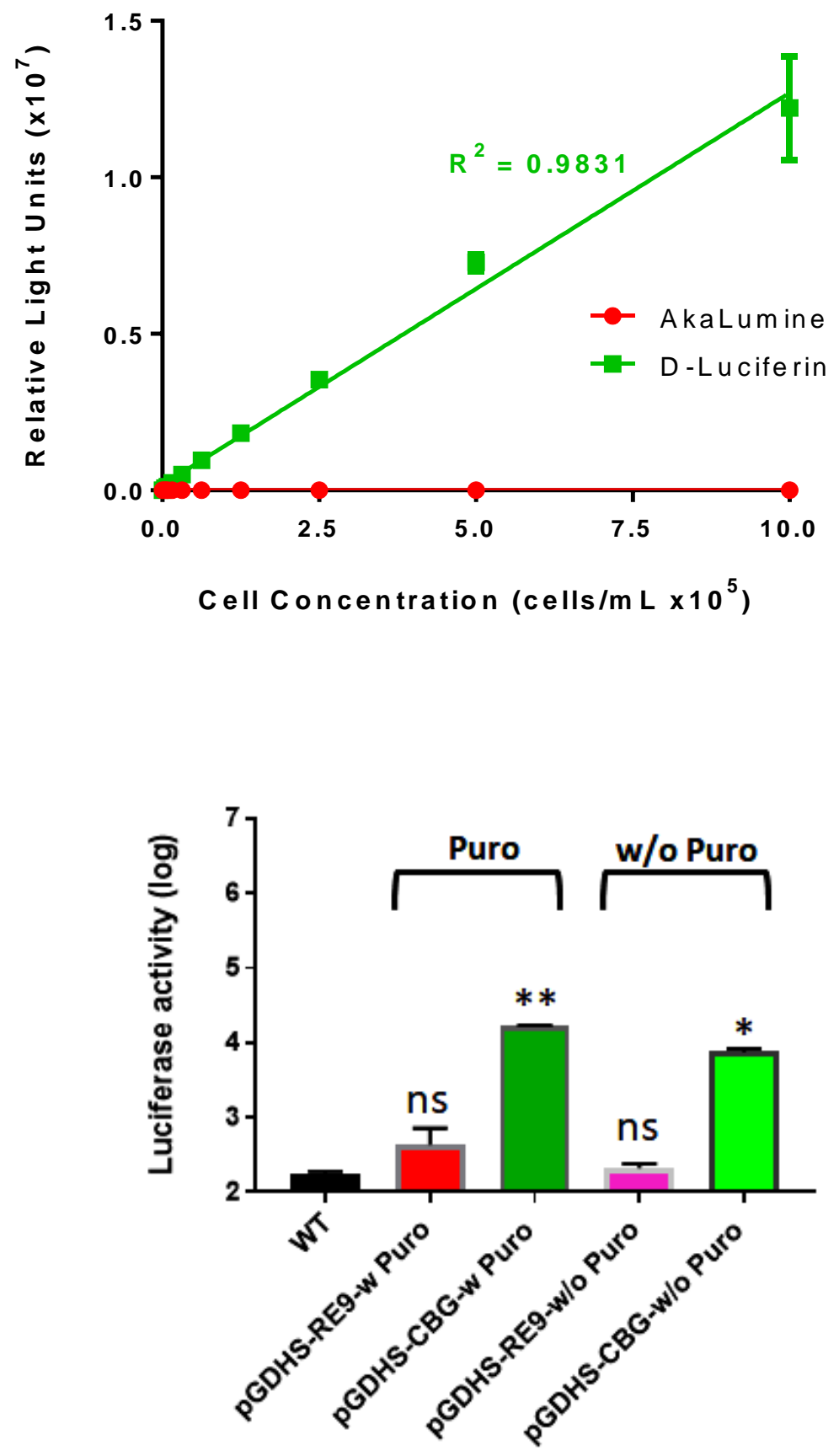

439 Figure 2: Parasite cell count vs relative light units (RLU). (a) The plot shows the linearity of 440 bioluminescence readouts from the G. lamblia WBC6:pGDHS::CBG99 strain. Readout follows 5 441 minutes of incubation at room temperature. AkaLumine showed no signal at any time point. (b) In 
442 vitro comparison of PpyRE9h-dependent and CBG99-dependent photon flux in the presence or

443 absence of selection antibiotic (puromycin) for four weeks. Level of significance is indicated by 444 ns=not significant, * $p \leq 0.001$ and ** $p \leq 0.0001$.

445

446

447

448

449

450

451

452

453

454

455

456

457

458

459

460

461

462

463

464

465

466 
Figure 3A: MetRS inhibitor screening of G. lamblia luciferase emitting trophozoites of

2FM MetRS Inhibitor Screen

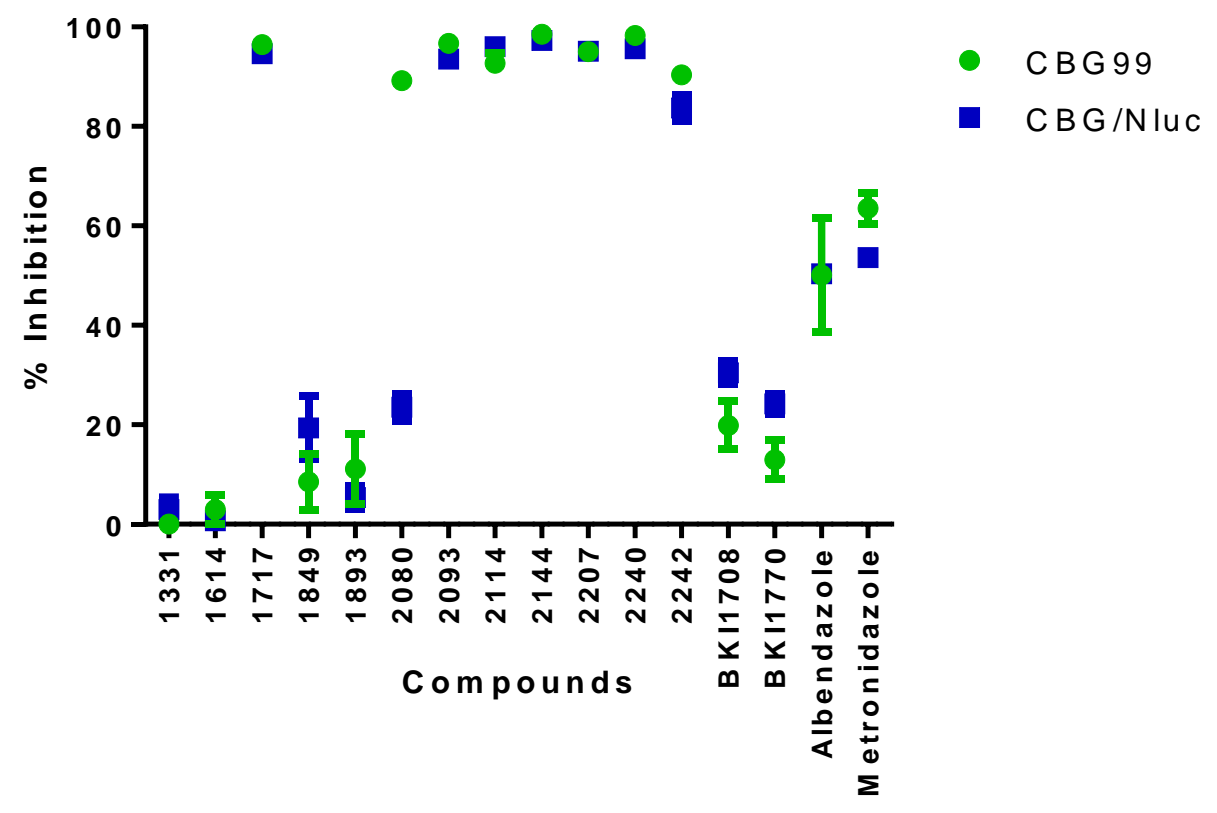

472 Figure 3B: A dose response plot of Compound 1717 inhibition of $G$. lamblia luciferase emitting 473 trophozoites WBC6:pGDHS::CBG99 versus WBC6:pGDHS::CBG/Nluc.

\section{Dose Response}

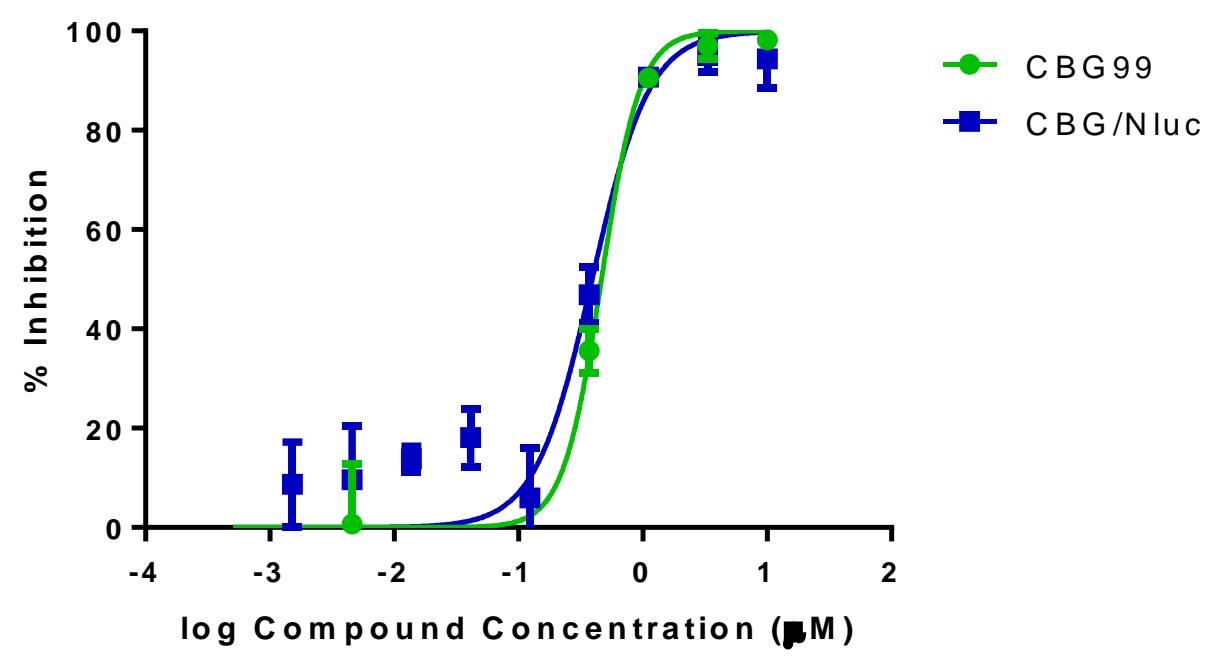


Figure 4: Radiance plot and Giardia mouse infections before and after treatment.

Radiance of Giardia

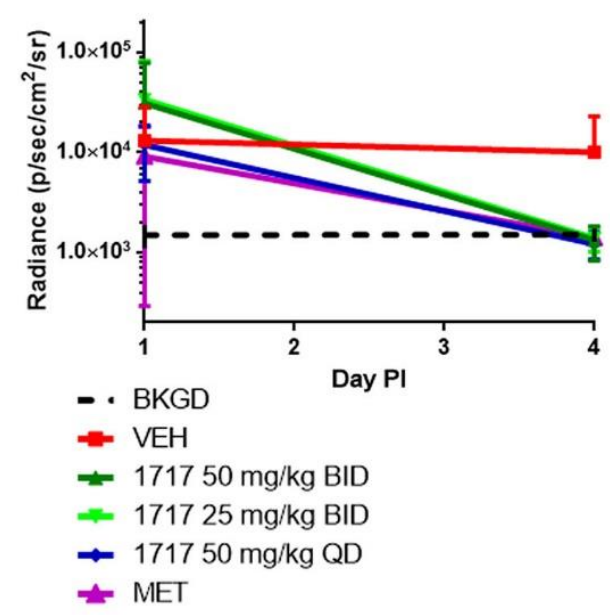

D1

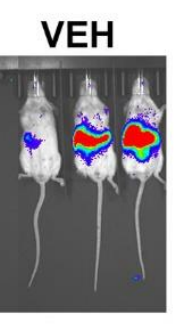

D4

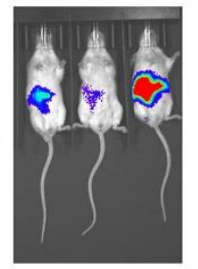

1717
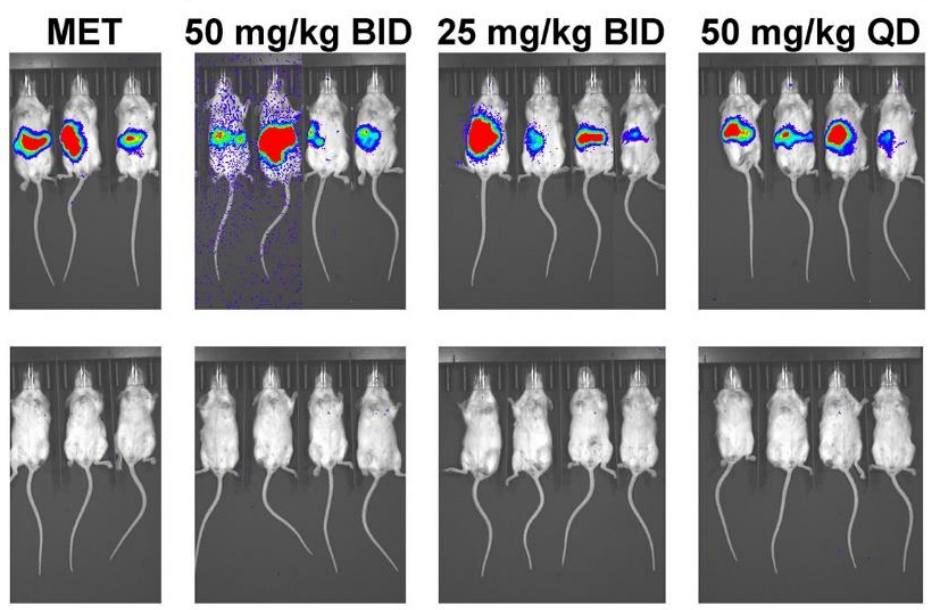

Figure 4: Radiance plot and Giardia mouse infections before and after treatment. These are noninvasive imaging of trophozoite growth in mice, 5 days post-infection (D1) with the G. lamblia WBC6:pGDHS::CBG99 strain before the start of treatment. Mice were treated with $50 \mathrm{mg} / \mathrm{Kg}$ Metronidazole BID (MET), compound 1717 dosed at $25 \mathrm{mg} / \mathrm{kg}$ or $50 \mathrm{mg} / \mathrm{kg} \mathrm{BID}$ and $50 \mathrm{mg} / \mathrm{kg}$ QD. Treatment duration was for 3 days. Mice Panel VEH were dosed with the dosing vehicle (3\% ethanol, $7 \%$ Tween 80 in normal saline) as a control. Imaging on day 8 post infection (D4) showed that 1717 and metronidazole cleared the infection relative to the untreated controls (VEH). The plot of the intensity/radiance data before therapy against days after start of treatment showed a significant drop relative to untreated controls. ${ }^{*}$ Note that images for some groups of mice on D1 have been cut together. This is due in part to differential intensity of the luminescence signal obtained from some mice hence differences in the length of time needed to acquire images that confirmed established infection. All images were taken during the same session and were scaled together.

\section{(1)}

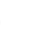




\section{Figure 5: G. lamblia WBC6:pGDHS::CBG99 versus G. lamblia WBC6:pGDHS::CBG/Nluc}

A

Trophozoite Growth Rate Comparison

501

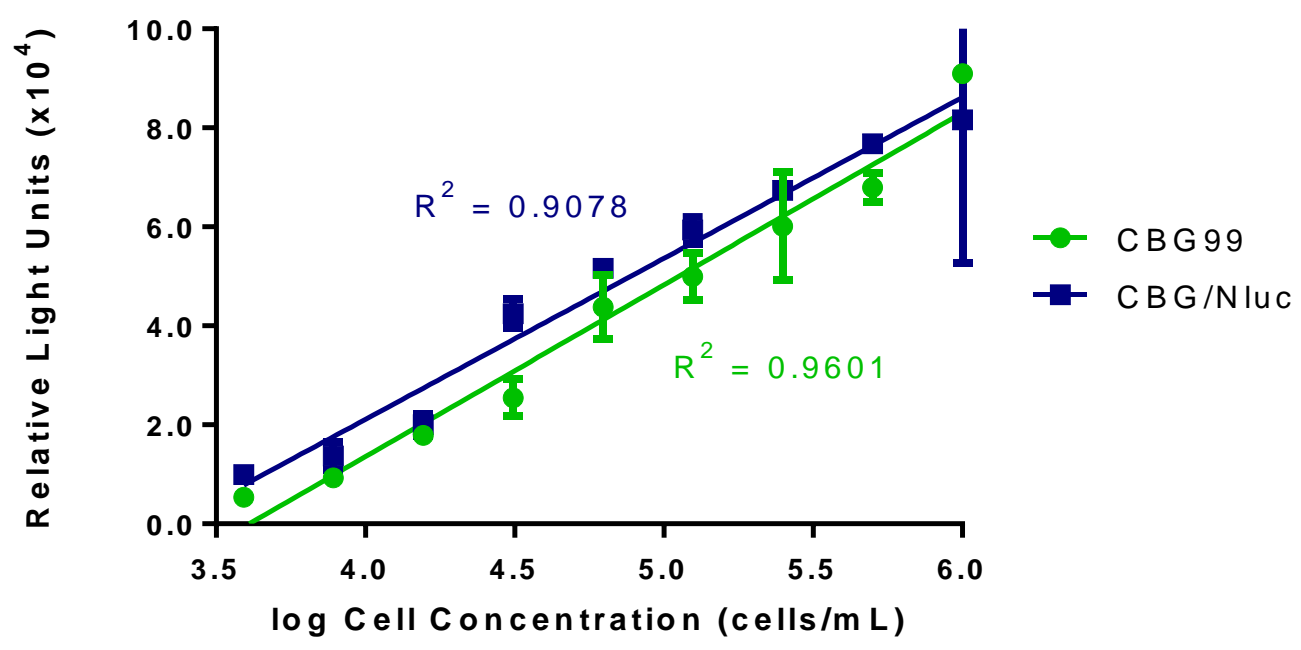

Cyst Lum inescence with NanoGlo

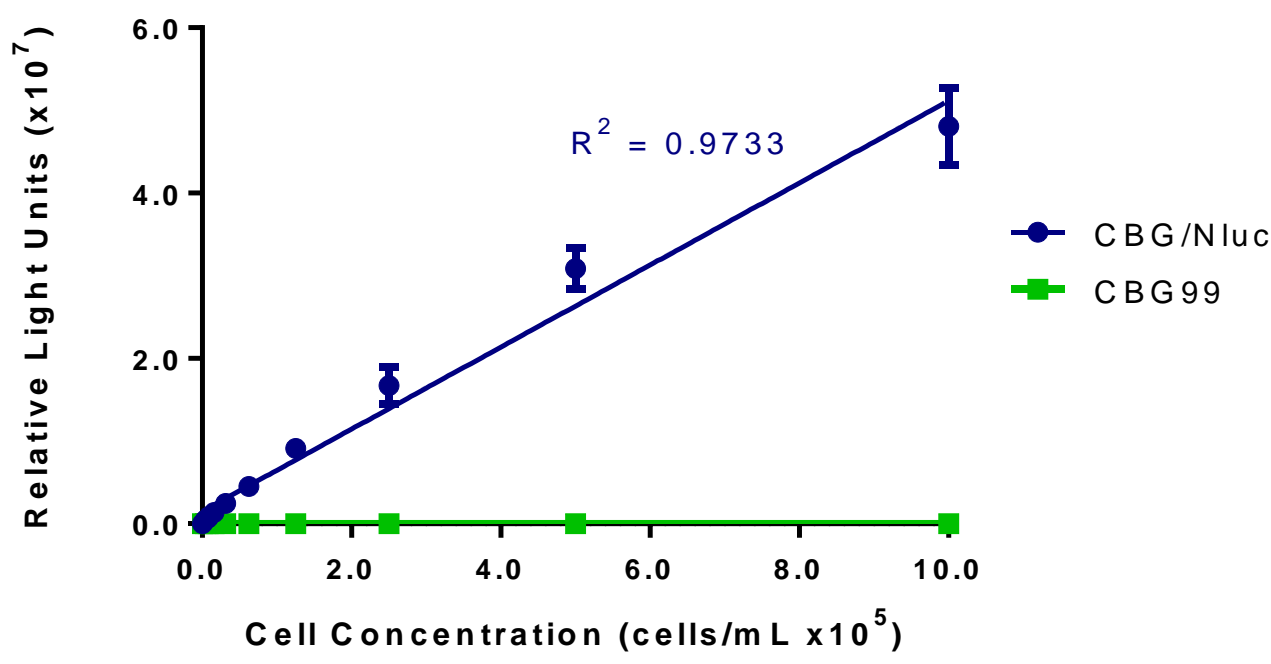

504 Figure 5: G. lamblia WBC6:pGDHS::CBG99 versus G. lamblia WBC6:pGDHS::CBG/Nluc, (a) A plot

G. lamblia WBC6:pGDHS::CBG/Nluc. The same serial concentrations of trophozoites from both strains were 
bioRxiv preprint doi: https://doi.org/10.1101/822924 this version posted October 29, 2019. The copyright holder for this preprint (which was not certified by peer review) is the author/funder, who has granted bioRxiv a license to display the preprint in perpetuity. It is made available under aCC-BY-ND 4.0 International license.

508 Plots of bioluminescence readouts from dual luciferase G. lamblia WBC6:pGHDS::CBG/Nluc cysts showed

509 a linear relationship to cell counts relative to no signal from the WBC6:pGDHS::CBG99 strain.

510

511

512

513

514

515

516

517

518

519

520

521

522

523

524

525

526

527

528

529

530

531

532 


\title{
References
}

534 1. Serradell MC, Saura A, Rupil LL et al. Vaccination of domestic animals with a novel oral vaccine

\begin{abstract}
prevents Giardia infections, alleviates signs of giardiasis and reduces transmission to humans. NPJ
\end{abstract} Vaccines 2016; 1: 16018.

2. Nabarro LE, Lever RA, Armstrong $M$ et al. Increased incidence of nitroimidazole-refractory giardiasis at the Hospital for Tropical Diseases, London: 2008-2013. Clin Microbiol Infect 2015; 21: 791-6. 3. $\quad$ Farthing MJ. Giardiasis. Gastroenterol Clin North Am 1996; 25: 493-515.

4. Tejman-Yarden N, Eckmann L. New approaches to the treatment of giardiasis. Curr Opin Infect Dis $2011 ; 24: 451-6$.

5. Lalle M. Giardiasis in the post genomic era: treatment, drug resistance and novel therapeutic perspectives. Infect Disord Drug Targets 2010; 10: 283-94.

6. Hill DR, Nash TE. Giardia lamblia. In: Mandell GL, Bennett JE, Dolin R, eds. Mandell, Douglas, and Bennett's principles and practice of infectious diseases. Philadelphia: Churchill Livingstone/Elsevier, 2010; 3527-34.

7. Muller J, Nillius D, Hehl A et al. Stable expression of Escherichia coli beta-glucuronidase A (GusA) in Giardia lamblia: application to high-throughput drug susceptibility testing. J Antimicrob Chemother 2009; 64: 1187-91.

8. Muller J, Ley S, Felger I et al. Identification of differentially expressed genes in a Giardia lamblia WB C6 clone resistant to nitazoxanide and metronidazole. J Antimicrob Chemother 2008; 62: 72-82.

9. Branchini BR, Ablamsky DM, Davis AL et al. Red-emitting luciferases for bioluminescence reporter and imaging applications. Anal Biochem 2010; 396: 290-7.

10. Hennessey KM, Rogiers IC, Shih HW et al. Screening of the Pathogen Box for inhibitors with dual efficacy against Giardia lamblia and Cryptosporidium parvum. PLoS Negl Trop Dis 2018; 12: e0006673.

11. Yee J, Mowatt MR, Dennis PP et al. Transcriptional analysis of the glutamate dehydrogenase gene in the primitive eukaryote, Giardia lamblia. Identification of a primordial gene promoter. J Biol Chem 2000; 275: 11432-9.

12. Jerlstrom-Hultqvist J, Stadelmann B, Birkestedt $\mathrm{S}$ et al. Plasmid vectors for proteomic analyses in Giardia: purification of virulence factors and analysis of the proteasome. Eukaryot Cell 2012; 11: 864-73. 13. Stefanic S, Morf L, Kulangara $C$ et al. Neogenesis and maturation of transient Golgi-like cisternae in a simple eukaryote. J Cell Sci 2009; 122: 2846-56.

14. Keister DB. Axenic culture of Giardia lamblia in TYI-S-33 medium supplemented with bile. Trans $R$ Soc Trop Med Hyg 1983; 77: 487-8.

15. Kuchimaru $\mathrm{T}$, Iwano $\mathrm{S}$, Kiyama $\mathrm{M}$ et al. A luciferin analogue generating near-infrared bioluminescence achieves highly sensitive deep-tissue imaging. Nat Commun 2016; 7: 11856.

16. Hall MP, Woodroofe CC, Wood MG et al. Click beetle luciferase mutant and near infrared naphthylluciferins for improved bioluminescence imaging. Nat Commun 2018; 9: 132.

17. Pham JK, Nosala C, Scott EY et al. Transcriptomic Profiling of High-Density Giardia Foci Encysting in the Murine Proximal Intestine. Front Cell Infect Microbiol 2017; 7: 227.

18. Read CM, Monis PT, Thompson RC. Discrimination of all genotypes of Giardia duodenalis at the glutamate dehydrogenase locus using PCR-RFLP. Infect Genet Evol 2004; 4: 125-30.

19. Boucher SE, Gillin FD. Excystation of in vitro-derived Giardia lamblia cysts. Infect Immun 1990; 58 : 3516-22.

20. Kane AV, Ward HD, Keusch GT et al. In vitro encystation of Giardia lamblia: large-scale production of in vitro cysts and strain and clone differences in encystation efficiency. $J$ Parasitol 1991; 77: 974-81.

21. Gil JS, Machado HB, Herschman HR. A method to rapidly and accurately compare the relative efficacies of non-invasive imaging reporter genes in a mouse model and its application to luciferase reporters. Mol Imaging Biol 2012; 14: 462-71.

22. Ur Rahman S, Stanton M, Casey PG et al. Development of a Click Beetle Luciferase Reporter System for Enhanced Bioluminescence Imaging of Listeria monocytogenes: Analysis in Cell Culture and Murine Infection Models. Front Microbiol 2017; 8: 1797.

23. Nakajima $Y$, Yamazaki $T$, Nishii $S$ et al. Enhanced beetle luciferase for high-resolution bioluminescence imaging. PLoS One 2010; 5: e10011. 
585 24. Xu T, Close D, Handagama W et al. The Expanding Toolbox of In Vivo Bioluminescent Imaging. 586 Front Oncol 2016; 6: 150.

587 25. Huang W, Hulverson MA, Choi R et al. Development of 5-Aminopyrazole-4-carboxamide-based 588 Bumped-Kinase Inhibitors for Cryptosporidiosis Therapy. J Med Chem 2019; 62: 3135-46. activity against Giardia intestinalis trophozoites. Antimicrob Agents Chemother 2015; 59: 7128-31.

27. Chen CZ, Kulakova L, Southall $N$ et al. High-throughput Giardia lamblia viability assay using bioluminescent ATP content measurements. Antimicrob Agents Chemother 2011; 55: 667-75.

28. Hennessey KM, Smith TR, Xu JW et al. Identification and Validation of Small-Gatekeeper Kinases as Drug Targets in Giardia lamblia. PLoS Negl Trop Dis 2016; 10: e0005107.

29. Byrd LG, Conrad JT, Nash TE. Giardia lamblia infections in adult mice. Infect Immun 1994; 62: 3583-5.

30. Wilke H, Robertson LJ. Preservation of Giardia cysts in stool samples for subsequent PCR analysis. J Microbiol Methods 2009; 78: 292-6.

31. Crannell ZA, Cabada MM, Castellanos-Gonzalez A et al. Recombinase polymerase amplificationbased assay to diagnose Giardia in stool samples. Am J Trop Med Hyg 2015; 92: 583-7.

32. Faghih O, Zhang Z, Ranade RM et al. Development of Methionyl-tRNA Synthetase Inhibitors as Antibiotics for Gram-Positive Bacterial Infections. Antimicrob Agents Chemother 2017; 61.

33. Zhang Z, Koh CY, Ranade RM et al. 5-Fluoroimidazo[4,5-b]pyridine Is a Privileged Fragment That Conveys Bioavailability to Potent Trypanosomal Methionyl-tRNA Synthetase Inhibitors. ACS Infect Dis 2016; 2: 399-404.

606 